\title{
Bessel-Function Integrals Needed for Two Classes of Physical Problems
}

\author{
By Charles E. McQueary and Lawrence R. Mack
}

1. Introduction. Expansions of functions either in the usual Fourier-Bessel series or in Dini series are often necessary in the solution of problems in physies or engineering for which the use of cylindrical coordinates is appropriate. Determination of the coefficients in these expansions requires the evaluation of integrals. In two quite different nonlinear physical problems [1], [2], (see also [3]) the need for integrals of products of three or four Bessel functions has been encountered. In the belief that these integrals may have application to other nonlinear problems involving Fourier-Bessel or Dini expansions, the authors present herein the values of the particular integrals needed for their own problems. In Section 2, integrals of products of four Bessel functions involving eigenvalues satisfying $J_{0}(x)=0$ are treated. In Section 3, integrals of products of three or four Bessel functions involving eigenvalues satisfying $J_{1}(x)=0$ are presented. A brief discussion of the computational procedure and of the accuracy of the results is given in Section 4 .

2. Integrals Involving Eigenvalues Satisfying $J_{0}(x)=0$. Let $K_{n}, n \geq 1$, be the positive eigenvalues, arranged in ascending order of magnitude, for which

$$
J_{0}\left(K_{n}\right)=0 .
$$

Let us introduce the shortened notation

$$
J_{m n} \equiv J_{m}\left(K_{n} r\right), \quad m=0 \text { or } 1,
$$

and define the integral operator $I(F)$ as

$$
I(F) \equiv \int_{0}^{1} r F(r) d r
$$

Fourier-Bessel expansions arising in the theory of oscillation of a circular membrane on a nonlinear Duffing-type foundation [1], [3] involve integrals $I(F)$ for which the functions $F(r)$ are products of four Bessel functions with arguments containing the eigenvalues (1). Integrals required for the second-order membrane solution for general values of the tension parameter and those required for the first-order solution for tensions near the particular first-order critical tension $T_{33}$ are listed in Table 1.

Each function with which we are concerned in this paper is sufficiently well behaved that the appropriate series expansion (Fourier-Bessel or Dini) is uniformly convergent to the function in the closed interval, $0 \leq r \leq 1$, [4, Chapter 18]. For each function $G(r)$ for which the first ten Fourier-Bessel coefficients are determinable from the integrals of Table 1 and for which the first ten Fourier-Bessel terms adequately represent the function, comparison of the successive partial sums which approximate the right side of the identity

Received October 19, 1966. 


$$
G(0)=2 \sum_{n=1}^{\infty} \frac{I\left(G J_{0 n}\right)}{J_{1}{ }^{2}\left(K_{n}\right)}
$$

provides an accuracy check.

Since Fettis [5] has presented values of $I\left(J_{0 i} J_{0 j} J_{0 k}\right)$ calculated by a different

TABLE 1

Integrals $I(F)$ of functions $F(r)$ for eigenvalues $K_{n}$ given by roots of $E q$. (1).

\begin{tabular}{|c|c|c|c|}
\hline$F(r)$ & $100 I(F)$ & $F(r)$ & $100 I(F)$ \\
\hline$J_{01}^{4}$ & 7.62101 & $J_{01}^{2} J_{05} J_{08}$ & -0.00261 \\
\hline$J_{01}^{3} J_{02}$ & 2.39034 & $J_{01}^{2} J_{05} J_{09}$ & 0.00068 \\
\hline$J_{01}^{3} J_{03}$ & 0.08820 & $J_{01}^{2} J_{05} J_{010}$ & $-0.00025^{-}$ \\
\hline$J_{01}^{3} J_{04}$ & -0.00810 & $J_{01}^{2} J_{06}^{2}$ & 0.83610 \\
\hline$J_{01}^{3} J_{05}$ & 0.00177 & $J_{01}^{2} J_{06} J_{07}$ & 0.41030 \\
\hline$J_{01}^{3} J_{06}$ & -0.00056 & $J_{01}^{2} J_{06} J_{08}$ & 0.01933 \\
\hline$J_{01}^{3} J_{07}$ & 0.00022 & $J_{01}^{2} J_{06} J_{09}$ & -0.00223 \\
\hline$J_{01}^{3} J_{08}$ & -0.00010 & $J_{01}^{2} J_{06} J_{010}$ & 0.00059 \\
\hline$J_{01}^{3} J_{09}$ & $0.00005^{-}$ & $J_{01}^{2} J_{07}^{2}$ & 0.71181 \\
\hline$J_{01}^{3} J_{010}$ & -0.00003 & $J_{01}^{2} J_{07} J_{08}$ & 0.35324 \\
\hline$J_{01}^{2} J_{02}^{2}$ & 2.80757 & $J_{01}^{2} J_{07} J_{09}$ & 0.01678 \\
\hline$J_{01}^{2} J_{02} J_{03}$ & 1.18217 & $J_{01}^{2} J_{07} J_{010}$ & $-0.00195^{+}$ \\
\hline$J_{01}^{2} J_{02} J_{04}$ & 0.05064 & $J_{01}^{2} J_{08}^{2}$ & 0.61973 \\
\hline$J_{01}^{2} J_{02} J_{05}$ & $-0.00535^{-}$ & $J_{01}^{2} J_{08} J_{09}$ & $0.31015^{+}$ \\
\hline$J_{01}^{2} J_{02} J_{06}$ & 0.00130 & $J_{01}^{2} J_{08} J_{010}$ & 0.01483 \\
\hline$J_{01}^{2} J_{02} J_{07}$ & -0.00044 & $J_{01}^{2} J_{09}^{2}$ & 0.54877 \\
\hline$J_{01}^{2} J_{02} J_{08}$ & 0.00019 & $J_{01}^{2} J_{09} J_{010}$ & 0.27646 \\
\hline$J_{01}^{2} J_{02} J_{09}$ & -0.00009 & $J_{01}^{2} J_{010}^{2}$ & 0.49239 \\
\hline$J_{01}^{2} J_{02} J_{010}$ & $0.00005^{-}$ & $J_{01} J_{02} J_{03}^{2}$ & 0.76184 \\
\hline$J_{01}^{2} J_{03}^{2}$ & 1.76106 & $J_{01} J_{03}^{3}$ & 0.49830 \\
\hline$J_{01}^{2} J_{03} J_{04}$ & 0.80034 & $J_{01} J_{03}^{2} J_{04}$ & 0.44991 \\
\hline$J_{01}^{2} J_{03} J_{05}$ & 0.03584 & $J_{01} J_{03}^{2} J_{05}$ & 0.53300 \\
\hline$J_{01}^{2} J_{03} J_{06}$ & $-0.00395^{+}$ & $J_{01} J_{03}^{2} J_{06}$ & 0.31108 \\
\hline$J_{01}^{2} J_{03} J_{07}$ & 0.00100 & $J_{01} J_{03}^{2} J_{07}$ & 0.01619 \\
\hline$J_{01}^{2} J_{03} J_{08}$ & $-0.00035^{-}$ & $J_{01} J_{03}^{2} J_{08}$ & -0.00212 \\
\hline$J_{01}^{2} J_{03} J_{09}$ & $0.00015^{+}$ & $J_{01} J_{03}^{2} J_{09}$ & 0.00061 \\
\hline$J_{01}^{2} J_{03} J_{010}$ & -0.00007 & $J_{01} J_{03}^{2} J_{010}$ & -0.00024 \\
\hline$J_{01}^{2} J_{04}^{2}$ & 1.28576 & $J_{02} J_{03}^{3}$ & 0.59604 \\
\hline$J_{01}^{2} J_{04} J_{05}$ & $0.60705^{-}$ & $J_{03}^{4}$ & 0.83760 \\
\hline$J_{01}^{2} J_{04} J_{06}$ & $0.02785^{-}$ & $J_{03}^{3} J_{04}$ & 0.44321 \\
\hline$J_{01}^{2} J_{04} J_{07}$ & -0.00314 & $J_{03}^{3} J_{05}$ & 0.28351 \\
\hline$J_{01}^{2} J_{04} J_{08}$ & 0.00081 & $J_{03}^{3} J_{06}$ & 0.21278 \\
\hline$J_{01}^{2} J_{04} J_{09}$ & -0.00029 & $J_{03}^{3} J_{07}$ & 0.16714 \\
\hline$J_{01}^{2} J_{04} J_{010}$ & 0.00013 & $J_{03}^{3} J_{08}$ & 0.12788 \\
\hline$J_{01}^{2} J_{05}^{2}$ & 1.01313 & $J_{03}^{3} J_{09}$ & 0.00781 \\
\hline$J_{01}^{2} J_{05} J_{06}$ & 0.48951 & $J_{03}^{3} J_{010}$ & -0.00123 \\
\hline$J_{01}^{2} J_{05} J_{07}$ & 0.02281 & & \\
\hline
\end{tabular}


TABLE 2

Integrals $I(F)$ of functions $F(r)$ for eigenvalues $K_{n}$ given by roots of $E q .(5)$.

\begin{tabular}{|c|c|c|c|}
\hline$F(r)$ & $100 I(F)$ & $F(r)$ & $100 I(F)$ \\
\hline$J_{01}^{3}$ & $2.85725^{+}$ & $J_{01} J_{06} J_{08}$ & 0.00879 \\
\hline$J_{01}^{2} J_{02}$ & 2.15858 & $J_{01} J_{06} J_{09}$ & -0.00115 \\
\hline$J_{01}^{2} J_{03}$ & $0.02145^{+}$ & $J_{01} J_{06} J_{010}$ & 0.00030 \\
\hline$J_{01}^{2} J_{04}$ & -0.00202 & $J_{01} J_{07}^{2}$ & 0.40048 \\
\hline$J_{01}^{2} J_{05}$ & 0.00040 & $J_{01} J_{07} J_{08}$ & 0.46119 \\
\hline$J_{01}^{2} J_{06}$ & -0.00011 & $J_{01} J_{07} J_{09}$ & 0.00777 \\
\hline$J_{01}^{2} J_{07}$ & 0.00004 & $J_{01} J_{07} J_{010}$ & -0.00103 \\
\hline$J_{01}^{2} J_{08}$ & -0.00002 & $J_{01} J_{08}^{2}$ & 0.35151 \\
\hline$J_{01}^{2} J_{09}$ & 0.00001 & $J_{01} J_{08} J_{09}$ & 0.40823 \\
\hline$J_{01}^{2} J_{010}$ & -0.00000 & $J_{01} J_{08} J_{010}$ & 0.00696 \\
\hline$J_{01} J_{02}^{2}$ & $1.35985^{-}$ & $J_{01} J_{09}^{2}$ & 0.31324 \\
\hline$J_{01} J_{02} J_{03}$ & $1.32595^{+}$ & $J_{01} J_{09} J_{010}$ & 0.36620 \\
\hline$J_{01} J_{02} J_{04}$ & 0.01766 & $J_{01} J_{010}^{2}$ & 0.28251 \\
\hline$J_{01} J_{02} J_{05}$ & $-0.00195^{-}$ & $J_{01}^{4}$ & 3.35702 \\
\hline$J_{01} J_{02} J_{06}$ & 0.00044 & $J_{01}^{3} J_{02}$ & 1.42132 \\
\hline$J_{01} J_{02} J_{07}$ & -0.00014 & $J_{01}^{3} J_{03}$ & 0.64857 \\
\hline$J_{01} J_{02} J_{08}$ & $0.00005^{+}$ & $J_{01}^{3} J_{04}$ & 0.01395 \\
\hline$J_{01} J_{02} J_{09}$ & -0.00002 & $J_{01}^{3} J_{05}$ & -0.00126 \\
\hline$J_{01} J_{02} J_{010}$ & 0.00001 & $J_{01}^{3} J_{06}$ & 0.00026 \\
\hline$J_{01} J_{03}^{2}$ & 0.91289 & $J_{01}^{3} J_{07}$ & -0.00008 \\
\hline$J_{01} J_{03} J_{04}$ & 0.96206 & $J_{01}^{3} J_{08}$ & 0.00003 \\
\hline$J_{01} J_{03} J_{05}$ & 0.01429 & $J_{01}^{3} J_{09}$ & -0.00001 \\
\hline$J_{01} J_{03} J_{06}$ & -0.00170 & $J_{01}^{3} J_{010}$ & 0.00001 \\
\hline$J_{01} J_{03} J_{07}$ & 0.00041 & $J_{01} J_{02} J_{11}^{2}$ & -0.08346 \\
\hline$J_{01} J_{03} J_{08}$ & -0.00014 & $J_{01} J_{03} J_{11}^{2}$ & -0.43773 \\
\hline$J_{01} J_{03} J_{09}$ & $0.00005^{+}$ & $J_{01} J_{04} J_{11}^{2}$ & -0.02113 \\
\hline$J_{01} J_{03} J_{010}$ & -0.00002 & $J_{01} J_{05} J_{11}^{2}$ & 0.00326 \\
\hline$J_{01} J_{04}^{2}$ & 0.69023 & $J_{01} J_{06} J_{11}^{2}$ & -0.00101 \\
\hline$J_{01} J_{04} J_{05}$ & 0.75603 & $J_{01} J_{07} J_{11}^{2}$ & 0.00042 \\
\hline$J_{01} J_{04} J_{06}$ & 0.01187 & $J_{01} J_{08} J_{11}^{2}$ & -0.00020 \\
\hline$J_{01} J_{04} J_{07}$ & -0.00148 & $J_{01} J_{09} J_{11}^{2}$ & 0.00011 \\
\hline$J_{01} J_{04} J_{08}$ & 0.00037 & $J_{01} J_{010} J_{11}^{2}$ & -0.00007 \\
\hline$J_{01} J_{04} J_{09}$ & -0.00013 & $J_{01} J_{11}^{3} / K_{1} r$ & 0.65784 \\
\hline$J_{01} J_{04} J_{010}$ & $0.00005^{+}$ & $J_{02} J_{11}^{3} / K_{1} r$ & -0.34902 \\
\hline$J_{01} J_{05}^{2}$ & 0.55573 & $J_{03} J_{11}^{3} / K_{1} r$ & -0.15058 \\
\hline$J_{01} J_{05} J_{06}$ & 0.62304 & $J_{04} J_{11}^{3} / K_{1} r$ & 0.00611 \\
\hline$J_{01} J_{05} J_{07}$ & 0.01011 & $J_{05} J_{11}^{3} / K_{1} r$ & -0.00207 \\
\hline$J_{01} J_{05} J_{08}$ & -0.00129 & $J_{06} J_{11}^{3} / K_{1} r$ & 0.00086 \\
\hline$J_{01} J_{05} J_{09}$ & 0.00033 & $J_{07} J_{11}^{3} / K_{1} r$ & -0.00042 \\
\hline$J_{01} J_{05} J_{010}$ & -0.00012 & $J_{08} J_{11}^{3} / K_{1} r$ & 0.00022 \\
\hline$J_{01} J_{06}^{2}$ & 0.46541 & $J_{09} J_{11}^{3} / K_{1} r$ & -0.00013 \\
\hline$J_{01} J_{06} J_{07}$ & 0.52999 & $J_{010} J_{11}^{3} / K_{1} r$ & 0.00008 \\
\hline
\end{tabular}


scheme for all distinct combinations of $i, j, k$ selected from among the integers 1,2 , 3 , we applied our method to these same ten integrals. In six cases the magnitudes of our results and of Fettis' are identical; however, the sign of $I\left(J_{01}^{2} J_{03}\right)$ was incorrectly shown as positive by Fettis, when actually $I\left(J_{01}^{2} J_{03}\right)$ is negative. Our results differ from those of Fettis in two cases by $1 \times 10^{-7}$ and in two cases by $2 \times 10^{-7}$.

Throughout this section, including Table $1, K_{n}$ have been the roots of $(1)$; in Section 3, however, $K_{n}$ will be the roots of (5).

3. Integrals Involving Eigenvalues Satisfying $J_{1}(x)=0$. Let $K_{n}, n \geq 0$, now be the eigenvalues for which

$$
J_{1}\left(K_{n}\right)=0, \quad K_{n} \geq 0,
$$

arranged in ascending order of magnitude beginning with $K_{0}=0$. Again we use the notation of (2) and (3). Dini series expansions arising in the theory of finiteamplitude axisymmetric gravity waves [2] involve integrals $I(F)$ for which the functions $F(r)$ are products of three or four Bessel functions with arguments containing the eigenvalues (5). Integrals required through the second nonlinear order in the gravity-wave solution are listed in Table 2.

Other integrals needed for gravity-wave theory are given in terms of those in Table 2 by the identities

$$
\begin{aligned}
I\left(J_{01}^{2} J_{11}^{2}\right) & =\frac{1}{3} I\left(J_{01}^{4}\right), \\
I\left(J_{11}^{3} / K_{1} r\right) & =\frac{3}{4} I\left(J_{01}^{3}\right),
\end{aligned}
$$

obtained from the results of Mack [6], and

$$
I\left(J_{11} J_{1 p} J_{0 n}\right)=\frac{{K_{1}}^{2}+K_{p}{ }^{2}-K_{n}{ }^{2}}{2 K_{1} K_{p}} I\left(J_{01} J_{0 p} J_{0 n}\right),
$$

obtained from the results of Fettis [5].

Values of $I\left(J_{11}^{2} J_{0 n}\right), 0 \leq n \leq 8$, and of $I\left(J_{11} J_{12} J_{0 n}\right), 0 \leq n \leq 6$, were computed both by direct integration and, by use of (8), from $I\left(J_{01}^{2} J_{0 n}\right)$ and $I\left(J_{01} J_{02} J_{0 n}\right)$, respectively; likewise, $I\left(J_{01}^{2} J_{11}^{2}\right)$ and $I\left(J_{11}^{3} / K_{1} r\right)$ were computed by direct integration and by use of (6) and (7), respectively. For each such integral the two values obtained differed by only about one-tenth the presumed error (see Section 4) in the individual integrals. An equally favorable comparison was obtained between the direct integration of $I\left(J_{01}^{3} J_{0 n}\right), 0 \leq n \leq 8$, and the values given by

$$
I\left(J_{01}^{3} J_{0 n}\right)=2 \sum_{m=0}^{\infty} \frac{I\left(J_{01}^{2} J_{0 m}\right) I\left(J_{01} J_{0 m} J_{0 n}\right)}{J_{0}{ }^{2}\left(K_{m}\right)},
$$

a special case of [6, Eq. (19), in which the subscript $p$ should read 0]. For each function $H(r)$ for which the first eleven Dini coefficients are determinable either directly or by use of (6), (7), or (8) from the integrals of Table 2 and for which the first eleven Dini terms adequately represent the function, comparison of the successive partial sums which approximate the right side of the identity

$$
H(0)=2 \sum_{n=0}^{\infty} \frac{I\left(H J_{0 n}\right)}{J_{0}^{2}\left(K_{n}\right)}
$$

provides an accuracy check. 
Although we have not yet found an analytical proof, the numerical result very strongly suggests that

$$
I\left(J_{01} J_{11}^{3} / K_{1} r\right)=\frac{1}{4} J_{0}^{4}\left(K_{1}\right) .
$$

4. Computational Procedure. The individual Bessel functions were generated by use of the well-known expression

$$
J_{m}(x)=\frac{1}{\pi} \int_{0}^{\pi} \cos (x \sin \theta-m \theta) d \theta, \quad m \geq 0,
$$

in which the generating integrals were evaluated by Gill's method [7]. Various step sizes were tried and the results were checked against standard tables [8]. It was found that 60 increments gave the best results, the errors in the values of $J_{0}(x)$ and $J_{1}(x)$ for $0 \leq x \leq 30$ being of the order of $10^{-8}$.

The desired integrals of products of Bessel functions were also evaluated by Gill's method, the number of increments used being increased with increases in the number of zeros which the product possesses in the range of integration. As a check on the accuracy of the procedure, those products of two Bessel functions whose integrals are known from the orthogonality relations were computed. In no case was the error as great as $4 \times 10^{-8}$. It is thus believed that for the majority of the integrals presented in Tables 1 and 2 the last digit shown is correct, although it is probable that the last digit is in error by one or even two units for some of the values. Other checks confirming the accuracy of the computations have already been mentioned in Sections 2 and 3.

No claim is made that the numerical-integration procedure followed is necessarily the most efficient one.

Acknowledgement. Use of the facilities of the Computation Center of The University of Texas is gratefully acknowledged.

Bell Telephone Laboratories, Inc.

Whippany, New Jersey

The University of Texas

Austin, Texas

1. L. R. MACK \& C. E. MCQUEARY, "Oscillations of a circular membrane on a nonlinear elastic foundation," J. Accoust. Soc. Amer. (to appear).

2. L. R. MAck, "Periodic, finite-amplitude, axisymmetric gravity waves," J. Geophys. Res., v. 67,1962 , pp. 829-843. MR 24 \#B1824.

3. C. E. McQueary \& L. G. Clark, "Nonlinear periodic modes of oscillation of elastic continua," J. Acoust. Soc. Amer. (to appear).

4. G. N. Watson, A Treatise on the Theory of Bessel Functions, 2nd ed., Cambridge Univ. Press, Cambridge; Macmillan, New York, 1944. MR 6, 64.

5. H. E. Fotris, "Lommel-type integrals involving three Bessel functions," J. Math. Phys., v. 36, 1957, pp. 88-95. MR 19, 771.

6. L. R. MACK, "Bessel-function identities needed for the theory of axisymmetric gravity waves," Math. Comp., v. 19, 1965, pp. 654-657. MR 33 \#2833.

7. S. A. GiLl, "A process for the step-by-step integration of differential equations in an automatic digital computing machine," Proc. Cambridge Philos. Soc., v. 47, 1951, pp. 96-108. MR 12, 538.

8. M. Abramowitz \& I. A. Stegun, (Editors), Handbook of Mathematical Functions, with Formulas, Graphs, and Mathematical Tables, National Bureau of Standards, Applied Math. Series, No. 55, U. S. Government Printing Office, Washington, D. C., 1964; reprint 1965. MR 29 \#4914; MR $31 \# 1400$. 